\title{
Eurozentrismus dekonstruieren. Zur Bedeutung postkolonialer Perspektiven auf schulische und außerschulische Bildungsangebote
}

\begin{abstract}
Zusammenfassung
Ausgehend von Erkenntnissen einer empirischen Erhebung $\mathrm{zu}$ Vorstellungen von Jugendlichen zur ungleichen globalen Verteilung von Weltkulturerbestätten werden verschiedene Aspekte diskutiert, um Eurozentrismus in Bildungsangeboten zu dekonstruieren. Dabei wird im Besonderen auf postkoloniale Perspektiven und die Bedeutung des Dekolonisierens eingegangen.
\end{abstract}

Schlüsselworte: Welterbe-Bildung, Eurozentrismus, postkoloniale Perspektiven, Dekolonisierung

\section{Abstract}

Based on findings from an empirical study on young people's perceptions of the inbalanced global distribution of cultural World Heritage sites, various aspects are discussed in order to deconstruct Eurocentrism in educational programmes. In particular, postcolonial perspectives and the importance of decolonising will be addressed.

Keywords: World Heritage Education, Eurocentrism, Postcolonial Perspectives, Decolonisation

\section{Einleitung}

Der Vorwurf des Eurozentrismus bzw. eurozentrischer Perspektiven wird zunehmend an Bildungsangebote bzw. Pädagogik und Erziehungswissenschaften adressiert (Knobloch, 2019). Als Eurozentrismus wird hier die vermeintlich selbstverständliche und bisweilen kaum reflektierte Auffassung Europas verstanden, die von einer epistemischen, kulturellen, ökonomischen und politischen globalen Hegemonie ausgeht. ${ }^{1}$ Bauer zeichnet nach, wie in Europa eine Vielzahl an Ideologien hervorgebracht wurde, „die vorgaben, die Welt eindeutiger als bisher erklären und den Menschen die einzig erstrebenswerte Zukunft vorzeichnen zu können. Durch den Kolonialismus und die europäische militärische und wirtschaftliche Überlegenheit verbreitete sich diese Weltdeutung auch global“ (2018, S. 42). So werden in Europa Tendenzen der Universalisierung eigentlich partikularer Positionen ausgemacht (Reuter, 2018, S. 273). Aus postkolonialen Perspektiven ist es notwendig, dass Europa vermeidet, die in seinem Namen verübte historische Gewalt in anders akzentuierter Weise zu wiederholen (Dhawan, 2016, S. 80). „Europa muss sich deswegen der Herausforderung stellen und wählen, ob es auf seinem früheren Weg des Anspruchs auf moralische, wirtschaftliche und militärische Übermacht gegenüber der nichteuropäischen Welt weitergehen will oder ob es der Herausforderung gewachsen ist, ein anderes Europa hervorzubringen, welches verantwortungsvoll und respektvoll gegenüber Differenz und Alterität ist“ (ebd.). Ein Schwerpunkt kann dabei darauf liegen, zu analysieren, wie lokale (europäische/westliche) Erkenntnistheorien eine Position einnehmen, die durch Institutionen wie Schulen und Universitäten sowie Organisationsformen wie Nationalstaaten als global und universell gesetzt werden (Andreotti, 2011, S. 3). So fordert auch Danielzyk in Bezug auf Globales Lernen, dass „der universale Charakter europäischer Entwicklungsimperative dekonstruiert und dadurch Europa als Norm dezentralisiert werden" (2013, S. 26).

Im folgenden Beitrag wird ausgehend von einer empirischen Erhebung zu Schüler/-innenvorstellungen zum Weltkulturerbe untersucht, inwiefern der Eurozentrismusvorwurf an schulische und außerschulische Bildungsangebote in Deutschland aufgrund der jeweils zugrunde gelegten erkenntnistheoretischen Bezüge gerechtfertigt zu sein scheint. Die Analyse erfolgt entlang kritischer Reflexionen zu Universalität und Pluriversalität. Dabei werden macht- und herrschaftskritische Perspektiven berücksichtigt, um Vorschläge zur Diskussion zu stellen, wie Bildungsangebote dekolonisiert werden können.

\section{Eindrücke aus der empirischen Forschung: Eurozentrismus in Schüler/-innenvorstel- lungen zum Weltkulturerbe}

Jugendliche bilden häufig eine Zielgruppe von Bildungsangeboten zur Vermittlung von Welterbe, jedoch wurden ihre Per- 
spektiven auf das Themenfeld bisher nicht aus didaktischer Sicht erforscht. Das vom Niedersächsischen Ministerium für Wissenschaft und Kultur geförderte Forschungsprojekt „Weltkulturerbe aus der Perspektive Jugendlicher - Vorstellungen, Bedeutungszuweisungen, Einstellungen und Werthaltungen im Kontext von Kulturbewusstsein und gesellschaftlicher Transformation" nahm sich diesem Desiderat an (Röll \& Meyer, 2018; Röll, 2021). Die Datenerhebung fand zwischen Mai 2017 und September 2018 statt. An der Studie partizipierten insgesamt 43 Schüler/-innen im Alter von 14-17 Jahren, aufgeteilt in zwölf Gruppen. Sechs Gruppen kamen aus Hannover und jeweils zwei Gruppen aus den Welterbestädten Alfeld, Goslar und Hildesheim. Jede der zwölf Gruppen nahm an zwei Fokusgruppendiskussionen teil, die im Anschluss mittels inhaltlich-strukturierter qualitativer Inhaltsanalyse (Kuckartz, 2016, S. 97) ausgewertet wurden. In den Fokusgruppen wurde u.a. erhoben, wie die teilnehmenden Jugendlichen die ungleiche globale Verteilung von Weltkulturerbestätten begründen (Röll \& Meyer, 2020; Röll, 2021).

Seit der Verabschiedung des Übereinkommens zum Schutz des Kultur- und Naturerbes der Welt (UNESCO, 1972) sind 897 Weltkulturerbestätten in die Welterbeliste aufgenommen worden. Davon liegen derzeit allein $47 \%$ in Europa (UNESCO, 2021). Diese Disparität ist u.a. auf das anfänglich sehr eurozentrisch geprägte Kulturerbeverständnis (UNESCO, 1994, S. 3), den langen und kostenintensiven Nominierungsprozess (UNESCO, 2015, S. 36), die Zusammensetzung des Welterbekomitees (Strasser, 2002, S. 242) sowie auf die politischen und wirtschaftlichen Interessen der Nominierungsund Mitgliedstaaten (Brumann, 2014, S. 2185; Meskell, 2018, S. 123) zurückzuführen.

In ihren Begründungsansätzen für die ungleiche Verteilung berufen sich die Teilnehmenden der Studie hingegen hauptsächlich auf demographische Entwicklungen, die Anzahl von als relevant empfundenen historischen Ereignissen, naturräumliche bzw. klimatische Bedingungen, kulturalistische $\mathrm{Zu}$ weisungen und entwicklungsbezogene Aspekte (Röll \& Meyer, 2020). Ausschlaggebend für die Annahmen der meisten Jugendlichen ist dabei die Vorstellung von Europa als einem herausragenden Hort der Kultur, Geschichte und des Fortschritts, die mit Vorstellungen zu Afrika und Südamerika sowie teilweise Asien kontrastiert werden. Insbesondere Afrika wird eine geringe historische Relevanz zugesprochen, herausgehoben wird lediglich seine Bedeutung in der menschlichen Evolutionsgeschichte. Dem Kontinent wird zudem wiederholt kein Beitrag zum Fortschritt zugesprochen. So ist z. B. Teilnehmerin G5_3 der Überzeugung, dass es „auch noch heute ,afrikanische Stämme' gibt, die noch richtig altmodisch in so Hütten wohnen." An diesem Beispiel wird auch das Verständnis der Jugendlichen des Begriffs „Entwicklung“ deutlich. Sehr präsent sind weiterhin geodeterministische Vorstellungen, die kulturelle Ausdrucksmöglichkeiten durch klimatische Bedingungen und Mangel an natürlichen Ressourcen beschränkt sehen (Röll \& Meyer, 2020).

Die hier skizzierten Sichtweisen sind u.a. geprägt von Ahistorisierung, Exotisierung, Hierachisierung von Kulturen, othering und Kulturalisierung - Mechanismen die nach den Analysen von u.a. Said (2017) und Bhabha (2011) als charakteristisch für den kolonialen Blick auf Länder im bzw. Men- schen aus dem sogenannten "Globalen Süden“ gelten (siehe dazu auch Kersting, 2011). Durch binäre Oppositionen im Sinne von "hier/dort" oder „fortschrittlich/unterentwickelt" wird in zahlreichen Aussagen das vermeintlich Andere konstruiert, wodurch „Starre und eine umwandelbare Ordnung, zugleich aber auch Unterordnung, Degeneriertheit und dämonische Wiederholung" (Bhabha, 2011, S. 97) festgeschrieben werden. Die durch die Jugendlichen vorgenommene Hervorhebung Europas als Zentrum der Weltgeschichte ist, wie bereits in der Einleitung herausgestellt, ein zentrales Merkmal von Eurozentrismus. Auch die daran anschließende Herabstufung anderer Kontinente, speziell Afrikas, hat Tradition. Sie lässt sich bereits in Werken von u.a. Kant (1968 [1802]) finden und kennzeichnet bis heute entsprechende Repräsentationen (siehe ausführlicher Eberth, 2019, S. 200ff.). So stellen beispielsweise Kiesel und Bendix in ihrer Analyse entwicklungspolitischer Plakate eine „vermeintliche Abwesenheit von Kultur im globalen Süden und die gleichzeitige Fokussierung auf Natur, Natürlichkeit und Ursprünglichkeit“" (2010, S. 486) fest.

Die kritische Aufarbeitung der ungleichen globalen Verteilung von Welterbe hat bisher noch keinen Eingang in die Welterbe-Bildung gefunden, vielmehr beschränken sich vorhandene Bildungsmaterialien (u.a. BLZ \& Zentrum Welterbe Bamberg, 2017; Deutsche Stiftung Denkmalschutz, 2010; Österreichische UNESCO-Kommission, 2007) auf die Vermittlung von Auswahlkriterien, involvierten Akteur/-innen oder den Nominierungsprozess als unumstößliche Fakten. Vergessen wird dabei, dass der den Welterbestätten zugeschriebene „außergewöhnliche universelle Wert“ (UNESCO, 2015, S. 17) keineswegs unumstritten ist: „Heritage value is not intrinsic; value is something that is attributed to an object, place or practice by particular people at a particular time for particular reasons" (Harrison, 2010, S. 25).

\section{Zur Ambivalenz des Universalitätsbegriffs in der Welterbediskussion}

Innerhalb der Welterbekonvention wird Universalität auf dreifache Weise aufgegriffen: „World Heritage is universal because it is of interest to humanity as a whole, because it embodies universal values, and because it is subject to universal practices" (De Cesari, 2010, S. 308). Der „außergewöhnliche universelle Wert" wird nicht nur innerhalb der critical heritage studies, sondern auch im Umfeld der UNESCO kontrovers debattiert. So kritisiert Byrne (1991, S. 232) die Annahme, dass weltweit alle Menschen ein Interesse am Schutz und Erhalt hätten, noch dazu am Erbe in anderen Staaten. Aufgrund der Schwierigkeit, Werte und Bedeutungen zu universalisieren, kommuniziert das Beratungsgremium ICOMOS (2008, S. 48) mittlerweile eine Herangehensweise, die Welterbestätten als herausragende Antworten auf universelle Herausforderungen betrachtet (z. B. Wasserversorgung, Handel, Mobilität, Stadtentwicklung). De Cesari problematisiert zudem die erzwungene weltweite Standardisierung von Erhaltungspraktiken: "More than just 'Western' per se [...] this mode is distinctively shaped by a capitalist and nationalist worldview, and bears the marks of a history of entanglements with the making of both colonial and bourgeois hegemony: heritage is a matter of civilization and distinction" (2010, S. 30). Trotz Bestrebungen der Bevorzugung „europä- 
ischer" Werte entgegenzuwirken und unterrepräsentierte Gruppen stärker zu fördern, konnten bisherige Maßnahmen das bestehende Ungleichgewicht nicht beheben (vgl. Steiner \& Frey, 2012, S. 32; Labadi, 2007, S. 159).

Der in der Welterbekonvention proklamierte Anspruch auf Universalität kann im Sinne Mignolos (2012, S. 200) somit zumindest teilweise als Universalisierung von regionalen Denkweisen verstanden werden sowie als Ausdruck von Kolonalität, d. h. der Kontinuität kolonialistischer Strukturen in heutigen Gesellschaften. Nach der von ihm skizzierten Matrix wirkt die Kolonalität der Macht durch Kontrolle von Ökonomie, Autorität, Natur und natürlicher Ressourcen, Geschlecht und Sexualität sowie Subjektivität und Erkenntnis (ebd., S. 50). Mit Bezug auf Quijano, sind diese verschiedenen Bereiche der kolonialen Matrix „durch eine Einbürgerung der okzidentalen Weltsicht miteinander verbunden, die den magischen Effekt produziert, glauben zu machen, die Welt entspräche dem, was in dieser Weltsicht von ihr ausgesagt wird. Die Entkopplung ist dringend notwendig und erfordert einen dekolonialen epistemischen Umsturz" (Mignolo, 2012, S. 57).

Zentraler Aspekt einer dekolonialen Bildung ist somit die Auseinandersetzung mit Mechanismen der Wissensproduktion und ihrer geopolitischen Einbettung. Dekolonialisierung „muss es darum gehen, die Beschränkungen zu analysieren, die die Orte des Denkens dem Denken selbst auferlegt haben. Dies sollte aber umgekehrt nicht dazu führen, dass der Ort des Denkens als determinierend für dieses Denken begriffen wird“ (Kastner \& Waibel, 2012, S. 33f.).

\section{Bildung dekolonisieren}

Die Ergebnisse der Studie von Röll und Meyer (2020) machen die Notwendigkeit einer Dekolonisierung von Bildungsangeboten deutlich. Die Erkenntnisse lassen darauf schließen, dass eurozentrische Denkmuster nicht nur in Bezug auf das konkrete Thema Weltkulturerbe bestehen, sondern, wie die erhobenen Daten zeigen, auch als Aspekt der Weltanschauung der an der Studie teilnehmenden Schüler/-innen verstanden werden können. Dies wird auch in verschiedenen Studien deutlich, die Schulbücher z. B. aus rassismuskritischer Perspektive oder hinsichtlich des vermittelten „Afrika“-Bildes untersuchen (u.a. Marmer, 2013; Marmer et al., 2015; im Überblick Eberth, 2019, S. 200ff.). Gerade die Themenfelder globale Disparitäten, Ursachen von Armut bzw. Entwicklungsdiskurse werden - neben vereinzelten innovativen Zugängen - im Schulunterricht bisweilen unsensibel und unkritisch besprochen (Mönter et al., 2016; Mönter \& Lippert, 2016). Ein konkretes Beispiel, wie sich dies im Bildungsbereich zeigt, ist die folgende Überschrift eines Artikels in der Zeitschrift "geographie heute“: "Wie Geographieunterricht Afrika verändern kann“ (Stein, 2019). Hier wird impliziert, dass Schulunterricht in Deutschland einen ganzen Kontinent verändern könne. Auch darin wird die Erhöhung der eigenen Stellung deutlich als jemand, der offenbar die Fähigkeit hat, Veränderungen zu vollbringen, über jemanden, der offenbar nicht aus eigener Kraft Veränderungen herbeiführen kann oder will. Daher bedarf es der klaren kritischen Reflexion eigener Haltungen und Handlungen in Vergangenheit und Gegenwart mit Blick auf die globale Hegemonie (definiert als die Macht, lokale ethnozentrische Perspek- tiven auf globaler Ebene durchzusetzen, zu normalisieren und zu naturalisieren).

Mignolo erkennt ein entsprechendes epistemisches Problem. „Der Gedanke von Zentren und Peripherien in der Ökonomie wurde auf die Bereiche der Erkenntnis und der Philosophie übertragen“ (2012, S. 48). Die wenig hinterfragte Verinnerlichung entsprechenden Eurozentrismus ist somit nicht nur durch Angebote aus dem Bereich der Welterbebildung zu begründen (diese nehmen ohnehin einen relativ geringen Anteil an den Themen der gängigen schulischen Kanones ein), sondern wirkt als Positionierung und Perspektive für Bewertungen auch auf andere Themenbereiche. So wird Kolonialität gerade auch u.a. in Bezug gesetzt zu einem eurozentrischen Kapitalismus und zur Globalisierung (Quijano, 2016, S. 23). Die Bedeutung einer Dekolonisierung von Bildung kann daher mit der hier skizzierten Studie zwar legitimiert werden, sie muss aber zwingend auch auf andere Bereiche als ausschließlich Weltkulturerbe bezogen werden. Bestehende Kolonialitäten zu benennen und zu kritisieren ist dabei zwar notwendig, reicht aber nicht aus, um Prozesse des Dekolonisierens zu praktizieren (Mignolo, 2012, S. 168). So wird es als äußerst herausfordernd erachtet, entsprechend Formen von Kolonialitäten zu überwinden (Abdi \& Schultz, 2012, S. 167); nicht zuletzt auch deshalb, weil es darum geht, „den Blick auf die Welt zu verändern, die vorherrschenden Wahrnehmungsmuster zu verändern" (Eribon, 2018, S. 65).

Zum besseren Verständnis werden die Begriffe „postkoloniale Perspektiven“ und „Dekolonisierung“ im Folgenden kurz skizziert. Auch nach dem formalen Ende des Kolonialismus sind alte und neue Formen von Imperialismus persistent (Dhawan, 2011; Harvey, 2005). So bleiben „Ideen von ,der Welt' oder ,dem Globalen ' [...] zutiefst mit dem Neokolonialismus verstrickt“ (Castro Varela \& Dhawan, 2015, S. 78). Insofern werden postkoloniale Perspektiven sowohl rückblickend auf die Zeit des Kolonialismus, als auch auf neokolonialistische Strukturen in der Gegenwart gerichtet. Postkolonialismus wird dabei verstanden „als eine Widerstandsform gegen die koloniale Herrschaft und ihre Konsequenzen“ (ebd., S. 16). So ist es die Intention postkolonialer Perspektiven, in eurozentrische Narrative zu intervenieren und hegemoniale Strukturen zu transformieren (ebd., S. 17). Ins Zentrum des Interesses sollten daher zunächst koloniale Denkmuster bzw. Weltbilder rücken sowie die Frage, welcher Einfluss einer kolonialen Prägung zur Entwicklung unserer Denk- und Weltbilder zukommt (vgl. Schröder, 2019, S. 35).

„Der ,Dekolonialismus` versteht sich als Intervention, als eingreifende Praxis: So wie Länder von Kolonialherren befreit wurden, gilt es Wissenschaften, Denken und Alltagspraxen von Kolonialität zu befreien“ (Kastner \& Waibel, 2012, S. 23). Es bedarf daher Bildungsangeboten, die dekoloniales Denken als infrage stellendes, zweifelndes, kritisches Denken verstehen (ebd., S. 30). Hinsichtlich der konkreten Problematik des Eurozentrismus kann dies verstanden werden als „praxisorientierte Haltung gegenüber dem als europäisch ausgemachten Denken“ (ebd., S. 31). Damit verfolgt dekoloniales Denken als Ziel eine Form des Lernens, mit der das koloniale Wissen gleichsam verlernt werden kann und „anderes“ Wissen zur Veränderung der Welt kennengelernt und diskutiert werden kann (ebd., S. 41). „Verlernen“ kann so als aktive kri- 
tisch-kollektive Intervention verstanden werden, mit dem Ziel, hegemoniale Wissensproduktionen zu hinterfragen und Demokratisierung zu fördern (Castro Varela \& Heinemann, 2016 u. 2017; siehe auch Capan et al., 2020). Die Schulung eines kritisch-reflexiven Denkens ist dazu notwendig, um einerseits nicht nur abstrakt zu reflektieren, sondern tatsächlich in eine reflexive Auseinandersetzung einzutreten, und andererseits „Verlerntes“ nicht bloß zu ersetzen, sondern im Sinne der Mündigkeit über kritisch-reflexive und emanzipatorische Fähigkeiten zu verfügen (siehe dazu Idahosa \& Bradbury, 2020). Dabei muss es Bildung „Subjekten ermöglichen, Unordentlichkeit und Irritation zu ertragen" (Castro Varela \& Heinemann, 2016) und diesen mitunter unbequemen Weg gehen: „engaging in critical reflexivity is a hard and messy process but to attain change we all, regardless of our identity and positionality, need to do the hard work of confronting our raced, classed, gendered and heteronormative privilege and our complicity in un/intentionally reproducing relations of domination" (Idahosa \& Bradbury, 2020, S. 34). Am Horizont des Dekolonisierungsprozesses wird „eine transmoderne, globale und vielfältige Welt“ (Mignolo, 2012, S. 67f.) ausgemacht, die bisweilen auch als Pluriversum bezeichnet wird (Kothari et al., 2018). Damit geht keineswegs die Absicht einher, etwa universelles Wissen im Kontext von Humanität und Menschenrechten zu relativieren oder gar zu marginalisieren. Vielmehr geht es um die Öffnung eines Dialogs unterschiedlichster erkenntnistheoretischer Traditionen (vgl. Mbembe, 2017; Khoo et al., 2020, S. 62f.), die Eingang in Bildungsangebote finden sollten. Es geht also darum, ,to pluralise epistemologies in our everyday lecturing work" (Khoo et al., 2020, S. 56). Wenngleich in anderen Zusammenhängen, so äußerte sich auch Arendt in ähnlicher Weise, sieht sie die Bedeutung des öffentlichen Raumes doch gerade in der „gleichzeitigen Anwesenheit zahlloser Aspekte und Perspektiven, in denen ein Gemeinsames sich präsentiert, und für die es keinen gemeinsamen Maßstab und keinen Generalnenner je geben kann. [...] Eine gemeinsame Welt verschwindet, wenn sie nur noch unter einem Aspekt gesehen wird; sie existiert überhaupt nur in der Vielfalt ihrer Perspektiven“ (2002, S. 56f.).

\section{Wie können dekolonisierte Bildungsange- bote aussehen?}

Das Anliegen einer Dekolonisierung von Bildung ist dezidiert nicht im Singular und insofern auch nicht als linearer Prozess zu verstehen. Kolonialitäten sind dabei intersektional verschränkt mit u.a. Rassismus, Klassismus, Seximus und Heteronormativität (Capan et al., 2020, S. 79). Weder kann eine Umsetzung der Dekolonisierung von Bildung also mit einfachen Akzentverschiebungen oder Interventionen gelingen, noch wird ein einfaches bzw. einseitiges Ergebnis angestrebt. „Die Zukunft wird weder von einem einzigen Lebensstil [...] regiert, noch wird sie von einem einzigen Befreiungs- und Dekolonialisierungsprojekt diktiert werden. [...] Eine Welt, in der viele Welten koexistieren können, lässt sich nur durch verteilte Arbeit und gemeinsame Ziele in der Vielfalt [...] erreichen " (Mignolo, 2012, S. 204f.). Es müssen Möglichkeiten eröffnet werden, „in einen pluriversalen Dialog unter Gleichen zu treten, in einen gemeinsamen Gang hin auf eine Welt, deren Ho- rizont „das freie Leben“ und nicht der „freie Markt" sein wird, „das gute Leben“ und nicht das „besser leben als der andere“ und letztlich eine kommunale und pluriversale Konstruktion, die keines Masterplans bedarf. Sollte es dennoch einen Masterplan geben, so wird er in einem kommunalen Prozess entwickelt werden und nicht von einer Elite, die weiß was gut für alle ist" (ebd., S. 207). Diese Ausführungen Mignolos verweisen auf drei Aspekte, die dem Vorhaben einer Dekolonisierung von Bildung als dienlich erscheinen:

(1) Eine monozentrische Weltsicht sollte ersetzt werden durch ein pluriverses Denken. Escobar (2018) entwirft das Pluriversum als Alternative zum Universum und damit die Vorstellung einer Welt, in die gleichsam viele Welten passen. Der Anerkennung der Werte von Vielfalt und Diversität als Charakteristika dieser Welt(en) kommt dabei zentrale Bedeutung zu. Dies ernst nehmend, wird ein Überdenken mancher Themen im schulischen Kontext notwendig, exemplarisch können Länderklassifikationen im Geographieunterricht genannt werden. Auch wenn anerkannt werden kann, dass einige dieser Ansätze durchaus als diversitätssensibel bezeichnet werden können, laufen diese doch häufig Gefahr, „im Eingehen auf Unterschiede diese nicht nur zu reproduzieren, sondern sie zugleich identitär festzuschreiben" (Hartmann et al., 2017, S. 15). Queertheoretisch inspirierte Perspektiven auf Bildung können in diesem Kontext ein hilfreicher Ansatz sein, da diese „Machtmechanismen (untersuchen), die Identitäten zuordnen, anordnen und kontrollieren [...]. [Sie] lassen Risse in der dominanten Ordnung als Umsetzungen von Entunterwerfung sichtbar werden, verweisen auf offene Möglichkeitsfelder“ (ebd., S. 17). Dabei wird „Differenz einerseits als Machteffekt von Klassifikationen und Kategorisierungen zurückgewiesen [...], andererseits [wird] darauf bestanden, Differenz zu artikulieren und sie - in ihrer Spezifik - anerkannt zu finden " (Engel, 2018, S. 339). Entsprechende Potenziale dieser Ansätze sollten stärker im größeren Zusammenhang der Analysen von Herrschafts- und globalen Ungleichheitsstrukturen gedacht werden (Castro Varela \& Rodríguez, 2000; Klapeer, 2015, S. 26; Engel, 2018, S. 340), „erweisen sich die dekolonialen Optionen [doch] als inspiriert von poststrukturalistischen und queeren Dekonstruktionen identitären und heteronormativen Denkens" (Kastner \& Waibel, 2012, S. 32).

(2) Zum Verständnis freien und guten Lebens: Diverse Verständnisse des „guten Lebens“ (Mignolo, 2012, S. 207) in unterschiedlicher Weise aufzugreifen, erweist sich als weiterer bildungsrelevanter Aspekt. Dies kann exemplarisch an Reflexionen zu ökonomischer Bildung konkretisiert werden. So sollten im Rahmen schulischer Bildung zunehmend Überlegungen jenseits des neoklassischen Mainstreams einbezogen, reflektiert und diskutiert werden (Braun, 2019). Ein konkretes Beispiel bieten Ansätze aus dem Bereich der Postwachstumsökonomien, denn „die Vielfalt von Lebensentwürfen wird dabei als ein zentrales Merkmal einer wünschenswerten Zukunft verstanden" (Schmelzer \& Vetter, 2019, S. 147; siehe auch Escobar, 2018, S. 139ff.). Dies könnte dazu beitragen, eurozentrische Perspektiven zu hinterfragen, da in entsprechender Analyse kapitalistischer Globalisierungsprozesse und von Entwicklungszusammenarbeit deutlich werden kann, dass die westlichen Industrieländer gleichsam vorgeben, „dass die ganze Welt nicht nur in demselben Maße, sondern auch in derselben 
Weise glücklich sein sollte wie sie“ (Mignolo, 2012, S. 126). Als mögliche Alternative thematisiert werden könnte z. B. das ecuadorianische Konzept des Buen Vivir („gutes Leben“; Roa, 2018). Dies wird dezidiert als plurales Konzept verstanden, „als verschiedene Arten des guten Zusammenlebens. Denn es geht nicht darum, die Tore für ein einziges, homogenes, nicht realisierbares gutes Leben zu öffnen, sondern vielmehr um das gute Miteinanderleben von Menschen in einer Gemeinschaft, von verschiedenen Gemeinschaften und von Individuen und Gemeinschaften einerseits und der Natur andererseits" (Acosta, 2017, S. 70; zum didaktischen Potenzial Rieckmann, 2017; Recknagel, 2018; unterrrichtspraktisch Meyer, 2018).

(3) Macht- und herrschaftskritsche Perspektiven: Die Bedeutung macht- und herrschaftskritischer Perspektiven ist ein weiterer Aspekt, auf den Mignolo hinweist. Im Kontext von Welterbe-Bildung kann dies in Bezug auf die Rolle der UNESCO verstanden werden. Hinsichtlich ökonomischer Strukturen und globaler Ungleichheitsverhältnisse kann der Einfluss des Kapitalismus und sog. Global Player kontextualisiert werden (siehe für das Beispiel Rosenzucht in Kenia; Eberth, 2020). Mignolo gibt im Besonderen aber den Impuls, über das Bildungssystem als solches nachzudenken. Im Anschluss kann etwa danach gefragt werden, wer vorgibt, was und wie in Schulen und an außerschulischen Lernorten gelernt werden soll. Welches sind die bestimmenden Institutionen und Personen und warum wird ihnen die Position zuerkannt, über Bildungsinhalte zu befinden? Welche Epistemologien sind Grundlage für die Themenkonstitution in den Schulfächern? Warum wird Kindern und Jugendlichen nicht stärker die Möglichkeit zuerkannt, über relevante und interessante Themen und Aspekte mitzuentscheiden? Diese Fragen deuten darauf hin, Themenkonstitution und Unterricht partizipativer zu gestalten sowie die Lernenden als Subjekte wahrzunehmen und Lernangebote von ihren Lebens- und Erfahrungswelten ausgehend zu gestalten - ähnlich, wie es für rassismuskritische Bildung von Schröder (2019) vorgeschlagen wird und im Bereich der Demokratiebildung in unterschiedlichen Formaten etabliert ist. Entsprechendes Potenzial liegt u.a. darin, situiertem Wissen mehr Raum zu geben. Gerade im Zusammenhang mit der Thematisierung globaler Ungleichheiten und des Entwicklungsbegriffs erscheint dies notwendig (Burchardt \& Tuider 2014, S. 391). Dies würde freilich erfordern, „dass das Wissensobjekt als Akteur und Agent vorgestellt wird und nicht als Leinwand oder Grundlage oder Ressource und schließlich niemals als Knecht eines Herrn, der durch seine einzigartige Handlungsfähigkeit und Urheberschaft von ,objektivem' Wissen die Dialektik abschließt" (Haraway, 1995, S. 93). Die Unterrrichtsmethoden des Thinking Through Geography-Ansatzes (Schuler et al., 2017) - um beim Beispiel des Geographieunterrichts zu bleiben - zeigen insgesamt gelungene Zugänge zur unterrichtspraktischen Aufbereitung situierten Wissens (Meyer, 2015, S. 186). Entsprechend sollten Akzente noch weiter verschoben werden: „Move from a universal view of knowledge, to an understanding that knowledge is socially, historically, culturally constructed. Similarly, move from a view of knowledge that is certain und unproblematic, to one that reflects a relational, multiperspectival understanding concepts such as culture, identity, space, place, interdependence, sustainability - knowledges, not knowledge; futures, not future; geographies, not geography; histories, not history“ (Martin, 2011, S. 220).

\section{Fazit: Implikationen für (außer-)schulische Bildungsangebote}

Postkoloniale Perspektiven können sinnstiftende Potenziale für schulisches Lernen bieten (vgl. Bechtum \& Overwien, 2017, S. 81). Daher sollten Forschungsarbeiten im Bereich postkolonialer Theorie stärker den Bereich Schule fokussieren; nicht zuletzt im Sinne von Wissenstransfer. Zudem sollten postkoloniale Perspektiven stärker als bisher Eingang in schulische Bildung finden. Folgende Orientierungen stellen wir in diesem Sinne als mögliche Vorschläge zur Diskussion:

- Neben der Betonung der Bedeutung von (Handlungs-) Kompetenzen, sollten vermehrt auch agency-Ansätze berücksichtigt werden, beziehen sich diese doch „stärker auf die Relationen und Beziehungsgefüge, in denen sich Handlungsmöglichkeiten entfalten, während die deutschen Begriffe Handeln als Kapazität eines Individuums fassen “ (Engel, 2015 S. 193). Dies kann auch im Kontext von Reflexivität gedacht werden, „denn das reflexive ,Ich“ (moi) entsteht immer ausgehend von seiner Einschreibung in die Geschichte (die Epochen, in denen man lebt) und in die Geographie (die Orte - und vor allem die Milieus - in denen die Leben verortet sind)“ (Eribon, 2018, S. 95).

- Curricula bzw. Lehrpläne und die fachdidaktische Themenkonstitution sollten kritisch hinsichtlich ihrer zugrundeliegenden epistemischen Bezüge hinterfragt werden. Damit einher geht eine Orientierung an Aspekten des Poststrukturalismus, die eine stärkere Berücksichtigung finden sollten, gerade weil Sprache eine diskursive Praxis ist, die ideologisch und instabil ist, was impliziert, dass Interpretationen der Welt die Welt oder die Realität selbst erschaffen (Andreotti, 2011, S. 87). Eine Reflexion von Worlding und Vermeidung (ab-)werter Begriffe wie „Entwicklungsländer“ kann so gelingen. Neben einer Diskussion über fachliche Konzepte ist dabei eine sprachliche Sensibilisierung essenziell, denn „Sprache ist ein ernstzunehmendes Machtinstrument, welches Diskriminierungspraxen wie Rassismus, aber auch sexistisches und klassenbasierte Exklusionsformen normalisiert" (Castro Varela \& Khakpour, 2019, S. 36).

- Subalterne Perspektiven (Spivak, 2008) sollten stärker berücksichtigt werden und im Zusammenhang mit der eigenen Positionalität zum Ausgangspunkt reflexiver Überlegungen werden. Dhawan betont die Bedeutung der „anhaltende(n) Hinterfragung der eigenen Komplizenschaft im fortwährenden zum Schweigen bringen marginalisierter Perspektiven" (2011, S. 31). Dies zeigt, dass vereinzelte gut gemeinte Initiativen oder Interventionen nicht ausreichend sind. Lediglich subalterne Perspektiven zu ergänzen, ist insofern unzureichend, denn „decolonisation involves both the coloniser and the colonised. It is not simply about finding ways of giving, voice' the hithero silenced and marginalised, but about challenging the epistemological basis of hegemonic thought" (Mercer et al., 2003, S. 428).

- Rolle und Arbeitsweisen von Organisationen (z.B. UNESCO) sollten kontextualisiert und hinsichtlich ihrer Positionalität reflektiert werden. Die Kritik an den institutionellen Strukturen und den zugrundeliegenden Denk- und Handlungsweisen des Welterbeprogramms 
bildet einen Schwerpunkt der critical heritage studies, ebenso wie Auseinandersetzung mit nationalstaatlichen Interessen bei der ,Schaffung 'von Kulturerbe (vgl. u.a. Meskell, 2018; Harrison, 2013; Byrne, 2008; Smith, 2006). So basiert laut Smith (2006, S. 11) der von der UNESCO angeführte authorizied heritage discourse (AHD) auf Vorstellungen von Nation und Klasse, technischem Wissen und Ästhetik, die in Europa beheimatet sind. Erbe wird so zu einem Gegenstand, über den lediglich Expert/-innen urteilen könnten.

- Kulturverständnisse sollten dezidiert zum Gegenstand von Bildungsprozessen gemacht und nicht nur implizit und unreflektiert mitgedacht werden; dabei sollte das eigene Kulturverständnis einer kritischen Reflexion unterzogen werden. Kultur und Bildung eigenen sich, um „Veruneindeutigungen und Regelüberschreitungen zu ermöglichen sowie Raum für Existenzen und Artikulationen jenseits der gerade gültigen Normalität zu schaffen. Sie dienten und dienen nicht nur für die Befriedung sozialer Spannungen im Interesse von Privilegierten, nichtnurzum Verweisaufdieangestammten sozialen Plätze, sondern auch als Vehikel für soziale Mobilität,alsRaumzurEntfaltungvonSelbstermächtigung und als Schauplatz und Instrument sozialer Kämpfe. [...] Kultur und Bildung so verstanden, sollen den Kampf gegen Ungleichheit unterstützen und Privilegien umverteilen, anstatt diese zu bestätigen und zu reproduzieren. Unverzichtbar für dieses Verständnis waren und sind Perspektiven aus dem Globalen Süden" (Mörsch, 2016, S. 180). Dazu bedarf es aber einer Abwendung von sog. „Paket-Kulturen“2 (Reuter, 2018, S. 270), die nach wie vor Grundlage einiger Bildungspläne darstellen. „Kultur ist vielmehr ein Fluss, der sich aus vielfältig synchron und diachron verknüpften Bedeutungen und Praktiken speist. Bedeutungen, Identitäten und Praktiken liegen dann nicht entweder in der einen oder der anderen Kultur, sie gehen durch sie hindurch und beziehen sie aufeinander - die Formel hierfür lautet ,hybrid'. Die Welt gleicht dann weniger einem Mosaik, dessen Steinchen die einzelnen Kulturen sind. Sie gleicht vielmehr einer Kulturmelange im Sinne einer wechselseitigen kulturellen Durchdringung globaler und lokaler Sinnbezüge, die in alltäglichen Praktiken mobilisiert und reproduziert wird“ (Reuter, 2018, S. 270; H. i. O.).

- Im Rahmen von Bildungsprozessen eine Ambiguitätstoleranz fördern: Thematisiert werden sollte „einerseits unser Umgang mit äußerer Vielfalt wie ethnischer Diversität oder eine Vielfalt an Lebensentwürfen, sowie andererseits auch unser Umgang mit vielfältigen Wahrheiten einer uneindeutigen Welt. Denn genau das ist unsere Welt: uneindeutig. Menschen sind ständig Eindrücken ausgesetzt, die unterschiedliche Interpretationen zulassen, unklar erscheinen, keinen eindeutigen Sinn ergeben, sich zu widersprechen scheinen, widersprüchliche Gefühle auslösen, widersprüchliche Handlungen nahezulegen scheinen. Kurz: Die Welt voll von Ambiguität" (Bauer, 2018, S. 12). Ambiguität wird insofern verstanden als ein Ansatz von Viel- bzw. Mehrdeutigkeit mit dem Potenzial, ,viele verschiedene
Bedeutungen und Assoziationen zu vermitteln" (ebd., S. 50).

Um Bildungsangebote in diesen Verständnissen zu rekontextualisieren, bedarf es sicherlich umfassender Anstrengungen der Bildungswissenschaften und Pädagogik, wie auch der jeweiligen Fachdidaktiken. Hinsichtlich entsprechender Gelingensbedingungen einer notwendigen dekolonialen epistemischen Pluriversifizierung macht Mignolo trotz ihres herausfordernden Charakters Mut: „Dieser Umsturz ist in verschiedenen Weltregionen bereits in Gang und bringt die Erkenntnis anderer Epistemologien, anderer Erkenntnis- und Verständnisprinzipien, anderen Ökonomien, Politiken und Ethiken mit sich“ (2012, S. 57).

\section{Anmerkungen}

1 Neben Europa gilt dies äquivalent für Nordamerika.

2 Mit diesem Begriff referiert Reuter auf ein Verständnis von Kulturen im Sinne des auf J. G. Herder zurückgehenden Kugelmodells. Kultur wird darin als gleichsam in sich geschlossen und nach außen zu anderen Kollektiven abgrenzbar verstanden, es wird eine vermeintlich eindeutige Differenz konstruiert (vgl. Reckwitz, 2001, S. 185). Ein derart akzentuiertes Kulturverständnis hat über den Kulturerdteil-Ansatz Eingang in einige Lehrpläne gefunden, so z. B. nach wie vor im Kerncurriculum Erdkunde an Gymnasien des Landes Niedersachsen.

\section{Literatur}

Abdi, A. A. \& Schultz, L. (2012). Recolonized Citizenship, Rhetorical Postcolonialities: Sub-Saharan Africa and the Prospects for Decolonized Ontologies and Subjectivities. In V. Andreotti \& L. M. de Souza (Hrsg.), Postcolonial Perspectives on Global Citizenship Education (S. 158-171). New York: Routledge.

Acosta, A. (2017). Buen Vivir: Die Welt aus der Perspektive des Buen Vivir überdenken. In Konzeptnetzwerk Neue Ökonomie \& DFG-Kolleg Postwachstumsgesellschaften (Hrsg.), Degrowth in Bewegung(en). 32 alternative Wege zur sozial-ökologischen Transformation (S. 70-83). München, oekom.

Andreotti, V. (2011). Actionable Postcolonial Theory in Education (Postcolonial Studies in Education). New York: Palgrave Macmillan. https://doi.org/10.1057/97802303 37794

Arendt, H. (2002). Vita activa oder vom tätigen Leben. München: Pieper.

Bauer, T. (2018). Die Vereindeutigung der Welt. Über den Verlust an Mehrdeutigkeit und Vielfalt. Stuttgart: Reclam.

Bechtum, A. \& Overwien, B. (2017). Kann postkoloniale Kritik Schule machen? Über ihre Grenzen und Potenziale für (entwicklungs-)politische Bildungsarbeit. In H.-J. Burchardt, S. Peters \& N. Weinmann (Hrsg.), Entwicklungstheorie von heute - Entwicklungspolitik von morgen (S. 59-84). Baden-Baden: Nomos. https://doi.org /10.5771/9783845267340-60

Bhabha, H. (2011). Die Verortung der Kultur. Tübingen: Stauffenburg.

BLZ: Bayerische Landeszentrale für politische Bildungsarbeit \& Zentrum Welterbe Bamberg (2017) (Hrsg.). welterbe elementar. München: BLZ.

Braun, B. (2019). Ökonomische Bildung im Geographieunterricht. Wo stehen wir? Welche Themen und Konzepte sollten künftig eine größere Rolle spielen? Praxis Geographie, 49(11), 4-9.

Brumann, C. (2014). Shifting Tides of World-Making in the UNESCO World Heritage Convention: Cosmopolitanisms Colliding. Ethnic and Racial Studies, 37(12), 2176-2192. https://doi.org/10.1080/01419870.2014.934261

Burchardt, H.-J. \& Tuider, E. (2014). Das vermachtete Subjekt. Feministisch-postkoloniale Perspektiven auf Entwicklung. In F. Müller, E. Sondermann, I. Wehr, C. Jakobeit \& A. Ziai (Hrsg.), Entwicklungstheorien. Weltgesellschaftliche Transformationen, entwicklungspolitische Herausforderungen, theoretische Innovationen: PVS Politische Vierteljahresschrift, Sonderheft 48, 381-404.

Byrne, D. (2008). Heritage Conservation as Social Action. In G. Fairclough, R. Harrison, J. Jameson Jnr. \& J. Schofield (Hrsg.), The Heritage Reader (S. 149-173). Milton Park \& New York: Routledge.

Capan, Z.G., Garbe, S. \& Zöhrer, M. (2020). How do we teach the world? Acto Academia, 52(1), 76-88. https://doi.org/10.18820/24150479/aa52i1/SP5

Castro Varela, M. \& Dhawan, N. (2015). Postkoloniale Theorie. Eine kritische Einführung. Bielefeld: transcript. https://doi.org/10.14361/9783839411483 
Castro Varela, M. \& Gutierrez Rodriguez, E. (2000). Queer Politics im Exil und in der Migration. In Quaestio (Hrsg.), Queering Demokratie. Sexuelle Politiken (S. 100112). Berlin: Quer.

Castro Varela, M. \& Heinemann, A.M.B. (2016): Ambivalente Erbschaften. Verlernen erlernen! Zwischenräume \#10, 12.

Castro Varela, M. \& Heinemann, A.M.B. (2017). „Eine Ziege für Afrika!“ Globales Lernen unter postkolonialer Perspektive. In: O. Emde, U. Jakubczyk, B. Kappes \& B. Overwien (Hrsg.), Mit Bildung die Welt verändern? Globales Lernen für eine nachhaltige Entwicklung (S. 38-54). Opladen: Barbara Budrich. https://doi.org/10.23 07/j.ctvdf0hpp. 5

Castro Varela, M. \& Khakpour, N. (2019). Sprache und Rassismus. In: B. Hafeneger, K. Unkelbach \& B. Widmaier (Hrsg.), Rassimuskritische politische Bildung. Theorien - Konzepte - Orientierungen (S. 33-44). Frankfurt a. M.: Wochenschau.

Danielzik, C.-M. (2013). Überlegenheitsdenken fällt nicht vom Himmel. Postkoloniale Perspektiven auf Globales Lernen und Bildung für nachhaltige Entwicklung. $Z E P$ - Zeitschrift für Internationale Bildungsforschung und Entwicklungspädagogik, 36(1), 26-33.

De Cesari, C. (2010). World Heritage and Mosaic Universalism. Journal of Social Archaeology, 10(3), 299-324. https://doi.org/10.1177/1469605310378336

Deutsche Stiftung Denkmalschutz (2010). denkmal aktiv. Arbeitsblätter für den Unterricht (3. Aufl.). Bonn: Deutsche Stiftung Denkmalschutz.

Dhawan, N. (2011). Transnationale Gerechtigkeit in einer postkolonialen Welt. In M. M. Castro Varela \& N. Dhawan (Hrsg.), Soziale (Un)Gerechtigkeit. Kritische Perspektiven auf Diversity, Intersektionalität und Antidiskriminierung (S. 12-35). Berlin: LIT.

Dhawan, N. (2016). Doch wieder! Die Selbst-Barbarisierung Europas. In M. Castro Varela, \& P. Mecheril (Hrsg.), Die Dämonisierung der Anderen, Rassismuskritik der Gegenwart (S. 73-83). Bielefeld: transcript. https://doi.org/10.14361/978383943 6387-005

Eberth, A. (2019). Alltagskulturen in den Slums von Nairobi. Eine geographiedidaktische Studie zum kritisch-reflexiven Umgang mit Raumbildern. (Sozial- und Kulturgeographie 30). Bielefeld: transcript. https://doi.org/10.14361/9783839447741

Eberth, A. (2020). Racist Roses? Ein kritischer Kommentar aus postkolonialer Perspektive zur Rosenzucht in Kenia. OpenSpaces - Zeitschrift für Didaktiken der Geographie, 02(02), 42-50.

Engel, A. (2015). Queere Politik der Paradoxie: Widerstand unter Bedingungen neoliberaler Vereinnahmung. In K. Walgenbach \& A. Stach (Hrsg.), Geschlecht in gesellschaftlichen Transformationsprozessen (S. 191-204). Opladen, Berlin u. Toronto: Barbara Budrich. https://doi.org/10.2307/j.ctvddzvpc.13

Engel, A. (2018). Geschlecht und Sexualität: Jenseits von Zweigeschlechtlichkeit und Heteronormativiät. In S. Moebius \& A. Reckwitz (Hrsg.), Poststrukturalistische Sozialwissenschaften (S. 330-346). Frankfurt a. M.: Suhrkamp.

Eribon, D. (2018). Grundlagen eines kritischen Denkens. Wien u. Berlin: Turia + Kant.

Escobar, A. (2018). Designs for the Pluriverse: Radical Interdependence, Autonomy, and the Making of Worlds. Durham u. London: Duke. https://doi.org/10.1215/9780822 371816

Haraway, D. (1995). Die Neuerfindung der Natur. Primaten, Cyborgs und Frauen. Frankfurt am Main: Campus.

Harrison, R. (2013). Heritage. Critical approaches. New York: Routledge.

Hartmann, J., Messerschmidt, A. \& Thon, C. (2017). Queering Bildung. In: dies. (Hrsg.). Queertheoretische Perspektiven auf Bildung. Pädagogische Kritik der Heteronormativität. Jahrbuch Frauen- und Geschlechterforschung in der Erziehungswissenschaft 13 (S. 15-28). Opladen, Berlin u. Toronto: Barbara Budrich. https://doi.org/ 10.3224/jfgfe.v13i1.02

Harvey, D. (2005). Der neue Imperialismus. Hamburg: VSA.

Idahosa, G.E. \& Bradbury, V. (2020). Challenging the way we know the world: overcoming paralysis and utilizing discomfort through critical reflexive thought. Acta Academia, 52(1), 31-53. https://doi.org/10.18820/24150479/aa52i1/SP3

Kant, I. (1968 [1802]). Kants Werke. Band: 9: Logik, Physische Geographie, Pädagogik. Berlin: de Gruyter.

Kastner, J. \& Waibel, T. (2012). Einleitung: Dekoloniale Optionen. In W. Mignolo, Epistemischer Ungehorsam. Rhetorik der Moderne, Logik der Kolonalität und Grammatik der Dekolonalität (S. 7-42). Wien u. Berlin: Turia + Kant.

Kersting, P. (2011). AfrikaSpiegelBilder und Wahrnehmungsfilter: Was erzählen europäische Afrikabilder über Europa? In P. Kersting \& K. W. Hoffmann (Hrsg.), AfrikaSpiegelBilder. Reflexionen europäischer Afrikabilder in Wissenschaft, Schule und Alltag (S. 3-10). Mainz: Geographisches Institut.

Khoo, S, Mucha, W., Pesch, C. \& Wielenga, C. (2020). Epistemic (in)justice and decolonization in higher education: experiences of a crosssite teaching project. Acta Academia, 52(1), 54-75. https://doi.org/10.18820/24150479/aa52i1/SP4

Kiesel, T. \& Bendix, D. (2010). White Charity: Eine postkoloniale, rassismuskritische Analyse der entwicklungspolitischen Plakatwerbung in Deutschland. PERIPHERIE, 30(120), 482-495.
Klapeer, C.M. (2015). Vielfalt ist nicht genug! Heteronormativität als herrschaftsund machtkritisches Konzept zur Intervention in gesellschaftliche Ungleichheiten. In F. Schmidt et al. (Hrsg.), Selbstbestimmung und Anerkennung sexueller und geschlechtlicher Vielfalt. Lebenswirklichkeiten, Forschungsergebnisse und Bildungsbausteine (S. 25-44). Wiesbaden: Springer VS. https://doi.org/10.1007/978-3-65802252-5_2

Knobloch, P.D.T. (2019). Global Citizenship Education und die Herausforderung epistemischer Dekolonialisierung. ZEP - Zeitschrift für internationale Bildungsfor schung und Entwicklungspädagogik, 42(4), 12-18. https://doi.org/10.31244/zep.20 19.04 .03

Kothari, A., Salleh, A., Escobar, A., Demaria, F. \& Acosta, A. (Hrsg.) (2018). Pluriverse: A Post-Development Dictionary. Delhi: Authors Up Front.

Kuckartz, U. (2016). Qualitative Inhaltsanalyse. Methoden, Praxis, Computerunterstützung (3. Aufl.). Weinheim u. Basel: Beltz Juventa.

Labadi, S. (2007). Representations of the nation and cultural diversity in discourses on World Heritage. Journal of Social Archaeology, 10(3), 299-324. https://doi.org/ $10.1177 / 1469605307077466$

Marmer, E. (2013). Rassismus in deutschen Schulbüchern am Beipiel von Afrikabildern. ZEP - Zeitschrift für internationale Bildungsforschung und Entwicklungspädagogik, 36(2), 25-31.

Marmer, E., Sow, P. \& Ziai, A. (2015). Der ,versteckte` Rassismus - ,Afrika im Schulbuch. In E. Marmer \& P. Sow (Hrsg.), Wie Rassismus aus Schulbüchern spricht. Kritische Auseinandersetzung mit,Afrika-Bildern und Schwarz-Weiß-Konstruktionen in der Schule. Ursachen, Auswirkungen und Handlungsansätze für die pädagogische Praxis (S. 110-129). Weinheim u. Basel: Beltz Juventa.

Martin, F. (2011). Global Ethics, Sustainability and Partnership. In G. Butt (Hrsg.), Geography, Education and the Future (S. 206-224). London: Continuum International Publishing Group.

Mbembe, A. (2017). Kritik der schwarzen Vernunft. Berlin: Suhrkamp.

Mercer, C., Mohan, G. \& Power, M. (2003). Towards a critical political geography of African development. Geoforum, 34, 419-436. https://doi.org/10.1016/S00167185(03)00045-9

Meskell, L. (2018). A Future in Ruins. UNESCO, World Heritage and the Dream of Peace. New York: Oxford University Press.

Meyer, C. (2015). Denken lernen mit Geographie. In S. Reinfried \& H. Haubrich (Hrsg.), Geographie unterrichten lernen. Die Didaktik der Geographie (S. 184-187). Berlin: Cornelsen.

Meyer, C. (2018). Verantwortung für Umwelt- und Klimaschutz im Geographieunterricht reflektieren - „Vier Blicke“ auf die Ölförderung im Yasuní-Nationalpark (Ecuador). In C. Meyer, A. Eberth \& B. Warner (Hrsg.), Diercke Klimawandel im Unterricht. Bewusstseinsbildung für eine nachhaltige Entwicklung (S. 190-206). Braunschweig: Westermann.

Mignolo, W. (2012). Epistemischer Ungehorsam. Rhetorik der Moderne, Logik der Kolonalität und Grammatik der Dekolonalität. Wien u. Berlin: Turia + Kant.

Mönter, L. O. \& Lippert, S. (2016): Entpolitisierte Entwicklungsländer? Gedanken zur Behandlung von Armutsursachen im Geographieunterricht. In A. Budke \& M. Kuckuck (Hrsg.), Politische Bildung im Geographieunterricht (S. 96-105). Stuttgart: Franz Steiner.

Mönter, L. O., Lippert, S. \& Gorges, A. (2016). Gemachte Armutsräume? Implikationen bei der Behandlung von Entwicklungsdisparitäten im geographischen Unterricht, vom Schulbuch zur Schülervorstellung. GW-Unterricht, 142/143(2-3), 71-79. https://doi.org/10.1553/gw-unterricht142/143s71

Mörsch, C. (2016). Stop Slumming! Eine Kritik kultureller Bildung als Verhinderung von Selbstermächtigung. In M. Castro Varela \& P. Mecheril (Hrsg.), Die Dämonisierung der Anderen, Rassismuskritik der Gegenwart (S.173-183). Bielefeld: transcript. https://doi.org/10.14361/9783839436387-012

ÖUK: Österreichische UNESCO-Kommission (Hrsg.) (2007). Welterbe für junge Menschen Österreich. Ein Unterrichtsmaterial für Lehrerinnen und Lehrer. Wien: ÖUK.

Quijano, A. (2016). Kolonialität der Macht, Eurozentrismus und Lateinamerika. Wien u. Berlin: Turia + Kant.

Recknagel, L. (2018). Buen Vivir, ein Thema für einen BNE-orientierten Geographie- (und Wirtschaftskunde) Unterricht? Analyse von Dokumenten mit unterrichtlichen Lernsettings. GW-Unterricht, 151(3), 34-42. https://doi.org/10.1553/ gw-unterricht 151 s34

Reckwitz, A. (2001). Multikulturalismustheorien und der Kulturbegriff. Vom Homogenitätsmodell zum Modell kultureller Interferenzen. Berliner Journal für Soziologie, 2, 179-200. https://doi.org/10.1007/BF03204013

Reuter, J. (2018). Globalisierung: Phänomen - Debatte - Rhetorik. In S. Moebius \& A. Reckwitz (Hrsg.), Poststrukturalistische Sozialwissenschaften (S. 263-276). Frankfurt a. M.: Suhrkamp.

Rieckmann, M. (2017). Bildung für nachhaltige Entwicklung in der Großen Transformation - Neue Perspektiven aus den Buen Vivir- und Postwachstumsdiskursen. In O. Emde, U. Jakubczyk, B. Kappes \& B. Overwien (Hrsg.), Mit Bildung die Welt 
verändern? Globales Lernen für eine nachhaltige Entwicklung (S. 147-159). Opladen, Berlin u. Toronto: Barbara Budrich. https://doi.org/10.2307/j.ctvdf0hpp.13

Roa, C. G. (2018). Buen Vivir, Pacha Mama, and the Defenders of Mother Earth. In R. Hembrom, T. Holthoff, G. Janecki et al. (Hrsg.), Learn2change. Transforming the World Through Education (S. 32-38). Hannover: Eigenverlag.

Röll, V. (2021). Zwischen, kollektivem Gedächtnis' und,nicht mehr benutzten Sachen - Weltkulturerbe aus der Perspektive Jugendlicher als Anstoß für eine kritisch-reflexive Welterbe-Bildung. Hannover: TIB Leibniz-Informationszentrum Technik und Naturwissenschaften Universitätsbibliothek. https://doi.org/10.15488/10902

Röll, V. \& Meyer, C. (2018). World Cultural Heritage from the Perspective of Young People - Preliminary Results of a Qualitative Study. In R. Amoeda, S. Lira, C. Pinheiro et al. (Hrsg.). Heritage 2018: Proceedings of the 6th International Conference on Heritage and Sustainable Development, Volume 2 (S. 1091-1102). Granada: Greenlines Institute.

Röll, V. \& Meyer, C. (2020). Vorstellungen von Jugendlichen über die ungleiche globale Verteilung von Weltkulturerbestätten - Didaktische Anregungen für eine kritisch-reflexive Welterbe-Bildung. GW-Unterricht, 157(1), 5-19.

Said, E. (2017). Orientalismus (5. Aufl.). Frankfurt a. M.: S. Fischer.

Schmelzer, M. \& Vetter, A. (Hrsg.). Degrowth/Postwachstum. Zur Einführung. Hamburg: Junius.

Schröder, B. (2019). Zugehörigkeit und Rassismus. Orientierungen von Jugendlichen im Spiegel geographiedidaktischer Überlegungen (Kultur und soziale Praxis). Bielefeld: transcript. https://doi.org/10.14361/9783839446942

Schuler, S., Vankan, L. \& Rohwer, G. (2017). Diercke Denken lernen mit Geographie. Methoden 1. Braunschweig: Westermann.

Smith, L. (2006) Uses of Heritage. New York: Routledge. https://doi.org/10.4324/9 780203602263

Spivak, G.C. (2008). Can the Subaltern Speak? Postkolonialität und subalterne Artikulation. Wien/Berlin: Turia + Kant.

Stein, C. (2019). Wie Geographieunterricht Afrika verändern kann. geographie heute, 343, 35-37.

Steiner, L. \& Frey, B. (2012). Correcting the Imbalance of the World Heritage List: Did the UNESCO Strategy Work? Journal of International Organizations Studies, 3(1), 25-40

Strasser, P. (2002). "Putting Reform Into Action" - Thirty Years of the World Heritage Convention: How to Reform a Convention without Changing its Regulations.
International Journal of Cultural Property, 11(2), 215-266. https://doi.org/10.1017/ S0940739102771427

UNESCO (1972). Übereinkommen zum Schutz des Kultur- und Naturgutes der Welt. Zugriff am 15.05.2020 https://www.unesco.de/sites/default/files/2018-02/UNESC O_WHC_Übereinkommen Welterbe_dt.pdf

UNESCO (1994). Expert Meeting on the "Global Strategy" and Thematic Studies for a Representative World Heritage List. WHC-94/CONF.003/INF.6. Paris.

UNESCO (2015). Richtlinien für die Durchführung des Übereinkommens zum Schutz des Kultur- und Naturerbes der Welt. WHC.15/01. Paris.

UNESCO (2020). World Heritage List Statistics. Zugriff am 24.04 .2020 https://whc. unesco.org/en/list/stat

\section{Dr. Andreas Eberth}

seit 2014 wissenschaftlicher Mitarbeiter im Arbeitsbereich Didaktik der Geographie am Institut für Didaktik der Naturwissenschaften der Leibniz Universität Hannover; Studium der Geographie, Germanistik und Bildungswissenschaften an der Universität Trier.

\section{Dr. Verena Röll}

ist seit 2020 wissenschaftliche Mitarbeiterin im Fachbereich Integrierte Verkehrsplanung am Institut für Land und Seeverkehr der TU Berlin; forschte von 2016 bis 2020 zu Weltkulturerbe aus der Perspektive Jugendlicher im Arbeitsbereich Didaktik der Geographie am Institut für Didaktik der Naturwissenschaften der Leibniz Universität Hannover; Studium der Stadt- und Regionalplanung, Urbanism, Historischer Urbanistik an der TU Berlin und der TU Delft. 